\title{
LES AFFINITÉS TAXONOMIQUES DE QUELQUES TRICHOPHYTON AFRICAINS, AGENTS DE TONDANTES MICROSPORIQUES OU TRICHOPHYTIQUES
}

\author{
Par J. BIGUet, G. COCHET, S. DEBLOCK, S. ANDRIEU et G. DUC
}

Depuis 1950 nous sont passées entre les mains de multiples souches de Dermatophytes africains en provenance de la Tunisie (1.263 souches) [14], du Cameroun (485) [12], de la Haute-Volta (93) [5], de la Côte-d'Ivoire (59 [15], du Togo (23) [inédit], de la Guinée (80) [1], du Sénégal (9) [5] et du Tehad (455) [6].

Parmi les Trichophyton isolés, quelques espèces présentent un intérêt particulier en raison de leur fréquence dans certains territoires; il s'agit tout spécialement de T. violaceum Bodin, 1902 $\left[28^{\mathrm{bix}}\right]$, de T. soudanense, Joyeux, 1912 [20] (1), T. gourvili Catanéi, 1933 [7], T. yaoundei Cochet et Doby-Dubois, 1957 [12], tous quatre agents de tondantes trichophytiques, et enfin de $T$. ferrugineum (Ota, 1922) [25], agent de tondantes microsporiques.

Dans la bibliographie, nous avons rencontré des prises de position ou des observations qui nous ont paru dignes d'attention. Les voici résumées :

$1^{\circ}$ Faut-il considérer $T$. soudanense comme un synonyme de $T$. ferrugineum (2), ou comme un mutant de cette dernière espèce ? La première

(1) La description de $T$. soudanense fut complétée par Joyeux en 1914 [21]. Initialement, nous avions accepté (Coutelen et coll., 1956) [14] le genre Langeronia créé pour ce dermatophyte par Vanbreuseghem en 1950 ; à la réflexion, nous devions l'abandonner (Cochet et coll., 1957) [12], sa distinction d'avec le genre Trichophyton Malmsten, 1843 , ne nous semblant pas reposer sur des bases suffisantes; on ne saurait naturellement guène plus partager l'opinion de Ansel (1957) [2] qui éloigne le genre Langeronia (Arthrosporés) des autres dermatophytes (Atractosporés). Contrairement aux observations de Vanbreuseghem reprises par Ansel, $T$. soudanense présente, en effet, de véritables aleuries qui étaient parfois très nombreuses dans certaines de nos souches, tout particulièrement parmi celles du Cameroun. D'autre part, les liens de parenté qui existent et que nous préciserons plus loin entre $T$. soudanense et $T$. gourvili dont le thalle présente une tendance beaucoup moins évidente à une abondante désarticulation (d'ailleurs non systématique, même lorsqu'on a affaire aux souches typiques de $T$. soudanense) sont una confirmation nouvelle de l'appartenance de $\%$. soudanense au groupe homogène des Dermatophytes et au genre Trichophyton.

(2) En réalité, ce serait évidemment $T$. ferrugineum qui tomberait en synonymie devant $T$. soudanense qui a l'antériorité. 
opinion fut soutenue par Salazar-Leite et ses collaborateurs en 1947 et $1950[30,31]$, et la seconde par le même auteur seul en 1958 [29]. Pour des raisons botaniques, l'espèce décrite par Ota, en 1922, sous l'appellation de $M$. ferrugineum [25], fut ensuite déplacée dans le genre Trichophyton par Talice en 1931 [34]. Ce transfert a été accepté par la grande majorité des auteurs. Tous cependant ne sont pas d'accord, Rivalier, en particulier [28], et quelques auteurs russes.

$2^{\circ}$ Vanbreuseghem et Van Brussel constataient en 1952 [38], sans d'ailleurs les confondre, l'étrange ressemblance d'un mutant violet de $T$, soudanense cultivé sur milieu à la terre, et des dermatophytes classés sous le nom de $T$. violaceum (3), T. gourvili ou $T$. pervesi (4).

$3^{\circ}$ En Afrique Noire, la présence de $T$. tonsurans a été signalée en Angola [32], en Haute-Volta [5], en, Côte-d'Ivoire [15]. Nous avons cru antérieurement pouvoir identifier à cette espèce : $T$. circonvolutum Sabouraud $1910\left[28^{\text {bis }}\right]$ du Dahomey, T. currii (Chalmers et Marshall 1914) [11] du Soudan anglo-égyptien, et un des Trichophyton innomine du Sénégal [5]. Dans le Nord de l'Afrique, $T$. tonsurans s'observe occasionnellement au Maroc espagnol [3], en Tunisie [14] et en Algérie [10], mais il serait banal en Tripolitaine [24] [5].

$4^{\circ}$ Pour Conant et coll. (1945 et 1954) [13], T. gourvili est synonyme de $T$. violaceum (qui a l'antériorité). C'est aussi l'avis de Langeron (1945) [22], qui considère $T$. gourvili comme étant vraisemblablement un jordanon indo-africain de $T$. violaceum.

$5^{\circ}$ Catanei (1939 [9] a étiqueté « $T$. violaceum neuf souches de Trichophyton camerounaises dont la pigmentátion est - « restée plutôt teinte pruneau ». Ce sont-des souches vraisemblablement identiques que l'un de nous et Doby-Dubois ont décrite au Cameroun en 1957 [12] sous la dénomination nouvelle de $T$. yaoundei.

A la lumière de notre expérience personnelle, voici les commentaires que nous inspirent ces diverses observations :

$1^{\circ}$ La lecture des textes de Salazar Leite et de ses collaborateurs $[30],[31],[32]$ sur la diagnose de $T$. ferrugineum en Angola et son identification à $T$. soudanense ne nous paraît pas convaincante. Il est exact que l'aspect macroscopique des colonies de l'une et de l'autre espèce peut prêter à confusion, d'autant qu'il est extrêmement variable ; Vanbreuseghem (5) avait déjà mis l'accent sur le polymorphisme de $T$. ferrugineum; nous avons retrouvé pareil phénomène pour $T$. soudanense, en sorte que les colonies serpigineuses de $T$. ferrugineum ressemblent à s'y méprendre à de nom-

(3) En 1955, Vanbreuseghem [37] a supprimé $T$. violaceum de cette liste.

(4) $T$. pervesi a été décrit par Catanei en 1937 [8].

(5) Dans la seconde édition du Précis de Mýcologie de Langeron, p. 550 [23]. 
breuses souches de $T$. soudanense et que certaines colonies faviformes de $T$. soudanense sont très voisines des colonies cérébroïdes de $T$. ferrugineum ; les deux espèces sont l'une et l'autre susceptibles de donner des colonies creuses (6).

Malgré ces convergences culturales ou morphologiques, l'identité des deux espèces ne peut, selon nous, être admise, au moins dans l'état actuel de nos connaissances, pour diverses raisons :

a) La lésion du cheveu est très différente ; pour notre part, nous avons toujours isolé $T$. ferrugineum de lésions microsporiques classiques et $T$. soudanense de lésions trichophytiques plus ou moins parfaitement constituées [dans les limites de notre expérience qui porte actuellement sur plus de 600 trichophyties déterminées par T. soudanense (Cameroun, Haute-Volta, Guinée Française, Côted'Ivoire, Sénégal, Tchad) et sur près de 70 microspories déterminées par T. ferrugineum (Cameroun, Haute-Volta) ]. Il semble qu'aucun fait contraire à nos observations n'ait jamais été publié (par Vanbreuseghem, entre autres, qui connaît bien ces deux Dermatophytes). Les auteurs portugais (qui insistent à juste titre sur les difficultés souvent réelles de la lecture des lésions des cheveux chez les Noirs) seraient done les seuls à admettre que $T$. ferrugineum soit susceptible de déterminer des lésions qui s'apparenteraient à des trichophyties. Ajoutons d'ailleurs qu'à notre connaissance - et quelles que soient les critiques qu'on puisse apporter sur d'autres aspects de la loi de spécificité (7) —, les Dermatophytes: déterminent des lésions pilaires caractéristiques (8); lorsqu'il nous arrive d'isoler, par exemple, $M$. audouini d'un Favus, nous ne pensons jamais que la loi de spécificité se trouve en défaut, mais qu'une sottise a été commise par l'un de nous ; les contrôles alors entrepris ont systématiquement justifié le bien-fondé de cette attitude.

b) Si l'aspect macroscopique des cultures peut être la source de confusions, l'image microscopique nous a permis jusqu'ici un diagnostic différentiel habituellement aisé ; alors qu'il est très diffi-

(6) Vanbreuseghem, en 1950 [36], avait toujours remarqué l'absence de cavité dans les colonies de $T$. soudanense qu'il avait isolées au Congo Belge.

(7) Cf. un article récent de Grigoraki [19].

(8) C'est là - nous en convenons - un aspect très restrictif de la loi de spécificité, mais il est parfaitement valable ; à notre connaissance, il ne serait en défaut qu'en ce qui concerne $T$. violaceum susceptible de déterminer éventuellement des lésions faviques; nous pensons que ces observations atypiques, statistiquement inexistantes, sont négligeables. 
cile, sinon impossible, de faire fructifier $T$. ferrugineum (9), même en utilisant des milieux favorables, $T$. soudanense présente très fréquemment, et même sur milieu de Sabouraud classique, au moins dans les souches que nous avons étudiées, des microconidies parfois nombreuses (cf. photo 1). D'autre part, le mycélium de T. ferrugineum ne présente jamais les hyphes secondaires orientées en sens inverse du sens de la croissance de l'hyphe principal, qui sont souvent très caractéristiques chez $T$. soudanense; il ne montre jamais non plus les organes nodulaires qui peuvent s'observer dans certaines souches de cette dernière espèce. Ne peuvent prêter à confusion, par conséquent, que certains aspects faviformes de $T$. soudanense, souvent localisés à quelques colonies d'une souche ou à la partie centrale de ces colonies.

L'identification de $T$. ferrugineum à $T$. soudanense parait donc dans ces conditions actuellement peu justifiée. Pour la même raison, il nous paraît très prématuré de considérer l'un de ces champignons comme un mutant de l'autre, et, en tout cas, nous serions très surpris que ce soit, conformément à l'opinion de Salazar Leite [29], l'espèce dont la morphologie microscopique est la plus riche qui dérive de l'espèce la plus pauvre. Au total, à lire les auteurs portugais et à l'examen des photographies qui illustrent leur travail de 1947 [31], nous rejoignons l'avis de Vanbreuseghem (1950) [36], à savoir que ces auteurs ont dû isoler, en réalité, $T$. soudanense, agent extrêmement répandu des trichophyties en Afrique Noire.

Quant à l'opinion des auteurs tels Rivalier, qui désirent replacer $T$. ferrugineum dans le genre Microsporum, elle mérite un examen approfondi. De nombreux auteurs acceptent, en effet, de réunir dans le même genre Trichophyton des espèces apparemment aussi différentes que, par exemple, T. mentagrophytes et T. soudanense d'une part et $T$. violaceum, $T$. verrucosum et $T$. schoenleini d'autre part, les premières produisant des colonies vivaces à morphologie microscopique riche ou relativement riche, les secondes des colonies faviformes, donc à pousse lente et à morphologie microscopique des plus pauvres. Si l'on admet un pareil polymorphisme et

(9) Impossible en ce qui concerne les souches que nous avons personnellement manipulées. C'est toutefois parce qu'il a obtenu, après un mois de culture sur crottin, des filaments de type Acladium portant de nombreuses aleuries, que Talice [39] a déplacé $M$. ferrugineum dans le genre Trichophyton. Son travail a été réalisé avec une seule souche qui lui fut expédiée à Paris de Montevideo, où elle avait été isolée d'un kérion typique ; l'auteur ne dit rien de la lésion pilaire microscopique. Nous ne sommes pas absolument convaincus qu'il ait eu réellement affaire à $M$. ferrugineum et, en tout cas, il serait souhaitable que les expériences de Talice puissent être répétées avec le même succès sur d'autres souches de $M$. ferrugineum. 
une telle évolution à l'intérieur d'un genre, pour quelle raison en nier la possibilité dans le genre voisin (10) ? Outre la nature de la lésion pilaire déterminée par $T$. ferrugineum, qu'on ne peut négliger - encore qu'il s'agisse là d'un fait qui, nous en convenons, ne suffit pas à emporter la conviction -, l'opinion de Rivalier se trouve renforcée par les résultats de quelques travaux russes. Des auteurs auraient réussi à induire la transformation de Trichophyton ferrugineum (pour eux, d'ailleurs, Microsporum ferrugineum) en $M$. lanosum (Sigalova) (1951) ou en un hybride voisin de cette dernière espèce (Zimérinov et coll., 1953) (11). Dans ces conditions, et si ces expériences se trouvaient confirmées, il y aurait peu de doute de l'appartenance réelle de Trichophyton ferrugineum au genre Microsporum.

$2^{\circ}$ L'opinion de Vanbreuseghem et coll. [37], [38], concernant les affinités de $T$. soudanense, dermatophyte jaune ou orange, et de $T$. gourvili ou de $T$. pervesi, tous deux à pigment violet, est des plus intéressantes (12), comme nous avons eu l'occasion de le constater en étudiant nos souches de $T$. soudanense et $T$. gourvili (13).

Nous avons d'abord confirmé l'observation de Vanbreuseghem et Van Brussel [38], sur l'apparition d'un «mutant» (14) violet de T. soudanense, lorsque celui-ci est cultivé sur milieu à la terre. Puis, nous avons obtenu spontanément un tel « mutant "sur milieu de Sabouraud classique à partir de deux souches en provenance l'une de Dakar, l'autre de Guinée Française. Enfin, en étudiant un important matériel du Tchad [6], nous avons isolé un premier lot de 397 souches de T. soudanense, dont 40 étaient partiellement pourvues de pigment violet ; 27 d'entre elles donnèrent plus ou moins rapidement des subcultures de $T$. soudanense typi-

(10) Lorsque Grigoraki écrit : «Achorion schoenleini, parasite du favus, est une espèce à morphologie semblable au Microsporum ferrugineum, et la séparation de ces deux espèces à deux genres différents est erronée » [19], il ne tient pas compte des phénomènes évolutifs de convergence morphologique éventuellement induits par les conditions du milieu sur lesquels Paldrok [27], de son côté, a le mérite de mettre l'accent (succession des stades florentiforme, rubriforme ef faviforme), encore qu'il aille, à notre avis, beaucoup trop loin dans les conclusions qu'il tire de son étude en réduisant à trois le nombre des espèces de dermatophytes valables, toutes trois groupées dans le seul genre Microsporum.

(11) Zimerinov et coll. ont cultivé $T$. ferrugineum trois mois et demi dans six filtrats de culture de $M$. lanosum. Nous n'avons malheureusement pas réussi à nous procurer leur texte, ni celui de Sigalova. Leurs travaux sont cités par Troyan en 1958 [35] dans une note malleureusement dépourvue de bibliographie.

(12) En ce qui concerne $T$. violaceum, l'analogie est plus discutable (cf. d'ailleurs la note 3 ).

(13) Malheureusement nous n'avons jamais eu la chance d'isoler $T$. pervesi. Récemment Catanei a eu l'obligeance de nous faire parvenir l'une de ses soushes, mais elle était très modifiée par le vieillissement.

(14) C'est nous qui mettons les guillemets. 
que et les 13 autres aboutirent chacune, suivant le point du prélèvement choisi pour le repiquage, à deux types de colonies, les unes du type $T$. soudanense, les autres du type $T$. gourvili.

Des cultures sur lames concernant, outre les colonies « mutantes », des souches de $T$. gourvili et de $T$. soudanense, les plus typiques possibles, n'ont pu fournir aucun caractère morphologique suffisamment constant pour permettre un diagnostic différentiel de certitude entre $T$. gourvili et $T$. soudanense typiques, et, a fortiori, entre ces derniers et les souches atypiques (cf. photographies 1, 2, $3,4)$. Tout au plus, semble-t-il, que, chez $T$. soudanense typique, les arthrospores, le mycélium buissonnant et les rameaux opposés au sens de ce mycélium, soient généralement plus abondants que chez $T$. gourvili typique, et que, chez cette dernière, au contraire, les organes nodulaires et les aleuries soient habituellement plus nombreux (15) ; mais il existe beaucoup d'exceptions à cette observation. Faute de pouvoir séparer $T$. soudanense et $T$. gourvili par la morphologie microscopique ou macroscopique, nous avons entrepris, dans l'intention d'apporter de nouveaux arguments qui permettent de mieux apprécier les statuts relatifs de ces espèces, une série de recherches complémentaires :

a), Catanéi (1933) [7] avait vu dans des cultures de $T$. gourvili sur grains d'orge quelques fuseaux comparables à ceux de $T$. soudanense. Dans les mêmes conditions culturales, nous avons observé chez l'une et l'autre espèce (typique ou atypique) des formations terminales qui ressemblent assez à un dessin illustrant le texte de Catanéi, mais nous hésitons à considérer ces formations comme d'authentiques fuseaux.

b) Les tests physiologiques de L. K. Georg [17] n'apportent aucun axgument valable pour distinguer $T$. soudanense et $T$. gourvili. II semble que les souches les plus typiques de ces deux champignons soient inhibées par la thiamine ; nous n'osons toutefois être trop affirmatifs, car les souches du Tchad, même typiques, nous ont donné une forte proportion de résultats aberrants (cf. photos 7 et 8 ) (nous avons soumis à ces tests : 65 souches de $T$. soudanense et $T$. gourvili typiques ou atypiques du Tchad, cinq $T$. soudanense typiques de Guinée et trois $T$. gourvili typiques de Haute-Volta). L'action inhibitrice de la thiamine ne se manifeste

(15) Vanbreuseghem et Van Brussel [38] avaient constaté l'apparition d'organes nodulaires chez un « mutant » violet de $T$. soudanense; nous avons retrouvé le même fait chez une souche « mutante 》 dakaroise du même dermatophyte (cf. photographies 5 et 6 ) qui au préalable n'en constituait pas. 
ni lors du repiquage des parties duveteuses de $T$. soudanense- $(16$ ? (habituellement composées d'hyphes sporifères), ni lors des cultures atypiques de $T$. soudanense (souches partiellement violettes) ou de T. gourvili (souches cireuses).

c) Au Tchad, la lésion du cheveu déterminée par T. soudanense ou T. gourvili typique est toujours neo-endothrix. Toutefois, dans la règle, la lésion occasionnée par le premier Dermatophyte semble aboutir plus difficilement au stade de maturité et le mycélium interne se fragmente en arthrospores rectangulaires, incapables de se transformer en spores rondes (classiques dans les trichophyties endothrix), auxquelles au contraire aboutissent souvent les infestations dues à T. gourvili. L'inoculation au cobaye, toujours positive, ne fournit, elle non plus, aucun caractère différentiel.

Les résultats de ces tests expérimentaux nous incitent à conclure qu'il n'existe aucune différence fondamentale entre $T$. soudanense et $T$. gourvili, que relient, au contraire, de nombreuses formes intermédiaires; le stade soudanense, jaune ou orangé ou rouille, constituerait un stade initial susceptible d'évoluer vers le stade gourvili violet, terminal. Nos observations sont en faveur d'une évolution orientée, mais progressive de ces champignons, et c'est pourquoi le nom de mutation ne nous paraît guère convenir à leur interprétation. Il semble que la transformation, réversible dans ses premiers stades, devienne ensuite irréversible, du moins dans les limites actuelles de notre expérience personnelle (en particulier, nous n'avons jamais vu un $T$. gourvili typique perdre, même partiellement, son pigment violet pour acquérir un pigment orangé). Ausși sommes-nous d'avis de faire tomber en synonymie $T$. gourvili Catanéi, 1933 devant $T$. soudanense Joyeux, 1912.

$3^{\circ}$ En ce qui concerne la diagnose de $T$. tonsurans dans les territoires d'Afrique, nous pensons utile d'attirer l'attention sur une confusion possible; il nous est arrivé plusieurs fois déjà d'identifier ce Dermatophyte en Haute-Volta [93] et en Guinée [1] ; au Tchad, au fur et à mesure des cultures entreprises sur le matériel abondant dont nous disposions, cette diagnose avait été posée initialement 13 fois pour des souches de Trichophyton blanes (17), qui

(16) Ce duvet blane existait chez 34 souclies seulement sur 410 souches de 7 . soudanense du Tchad (formes atypiques comprises, formes gourvili typiques exceptées); en grattant ce duvet, on voit le pigment orangé qui est plus ou moins abondant.

(17) Les colonies correspondant à ces souches, recouvertes d'un fin duvet blanc de neige et dépourvues de pigment même dans la profondeur de la culture, présentaient de très nombreuses aleuries en thyrses simples ou en grappes (photo $10)$; elles n'ont fourni ni intrications buissonneuses, ni vrilles, ni fuseaux en primoculture. 
ressemblaient à un $T$. tonsurans un peu atypique et plus vivace que celui des souches métropolitaines (photo 9). En fait, l'étude ultérieure attentive de ces souches nous a incités à modifier notre détermination ; nous avons en effet réalisé, outre l'examen de leurs cultures et de leurs subcultures sur milieux classiques, un certain nombre d'épreuves (tests physiologiques de L.-K. Georg, cultures sur fragments de cheveux in vitro et sur milieu à l'extrait de cheveux humains, inoculation au cobaye). Aucune d'elles n'a permis d'écarter le diagnostic de T. tonsurans. Cependant, l'épreuve effectuée sur les milieux de Georg avait montré que ces Trichophyton blancs étaient systématiquement autotrophes et indifférents à l'adjonction de thiamine (ou d'inositol), alors que, suivant Georg [17], la croissance de $T$. tonsurans est stimulée par la thiamine ; mais deux souches étiquetées $T$. tonsurans, dans le laboratoire de Rivalier, à l'Hôpital St-Louis (Paris), n'étaient pas non plus influencées par la thiamine, ce qui incite à la prudence quant à l'interprétation des tests de Georg (18). Mais aucune des recherches complémentaires n'a non plus révélé une quelconque différence de comportement d'avec $T$. soudanense au stade soudanense ou gourvili; d'autre part, les subcultures de neuf de ces souches se sont pigmentées en jaune ou en orangé, et certaines autres ont présenté en cultures sur lames des images de mycélium buissonnant assez caractéristique de $T$. soudanense. Si l'on ajoute à ces faits que nous n'avons observé au Tchad aucune souche typique de $T$. tonsurans, qu'au contraire $T$. soudanense y était représenté par de très nombreuses souches typiques et atypiques, que la lésion pilaire humaine déterminée par les Trichophyton blancs ne se distinguait en rien de celle qu'occasionne $T$. soudanense au stade soudanense, les arguments nous paraissent nombreux qui incitent à considérer ces Trichophyton blanes comme des formes apigmentées et atypiques de $T$. soudanense. Et il est également assez vraisemblable que les souches de Haute-Volta et de Guinée, que nous avons antérieurement étiquetées $T$. tonsurans et que malheureusement nous n'avons pas conservées, se rattachaient également à la même espèce. Par ailleurs, rien ne prouve que $T$. circumvolutum, d'origine dahoméenne et décrit très incomplètement par Sabouraud $\left[28^{\text {bis }}\right]$, n'était pas en réalité un $T$. soudanense faviforme et apigmenté. Egalement sujette à caution nous apparait la diagnose de $T$. tonsurans ( $T$. acuminatum), trois fois posée par Salazar Leite et Ré en Angola [32], et

(18) Précisons que nos milieux ont été préparés suivant la formule et avec les mêmes produits (D.I.F.C.O.) que ceux de l'auteur américain, et que nos expériences ont toujours été plusieurs fois répétées sur des souches soigneusement purifiées. 
que n'étaye d'ailleurs aucune description précise. Et peut-être même avons-nous eu tort antérieurement d'identifier, à $T$. tonsurans, $T$. currii (Chalmers et Marshall, 1914) [11], dont il serait en fait souhaitable de refaire une étude soigneuse avant de se prononcer sur sa diagnose (19). Enfin, il n'est pas inutile de souligner que les déterminations de $T$. tonsurans ou d'espèces satellites dans le Nord de l'Afrique furent appliquées souvent à des souches très atypiques. Pourtant, l'absence habituelle de $T$. soudanense dans cette partie du continent africain les rend infiniment moins suspectes qu'en Afrique Noire.

$4^{\circ}$ Quant à l'identification de $T$. gourvili à $T$. violaceum, elle prête largement à discussion. La ressemblance entre les formes typiques de ces deux espèces est en réalité assez mince et réduite généralement à la couleur violette du pigment. $T$. gourvili est beaucoup plus vivace que $T$. violaceum et ses caractères microscopiques le rapprochent bien plus, comme nous l'avons vu, de $T$. soudanense que de $T$. violaceum, qui est faviforme, très généralement dépourvu d'aleuries, et ne montrant jamais, à notre connaissance, d'hyphes à orientation inversée. Cependant, nous avons observé au Tchad des $T$. gourvili à pousse lente qui, macroscopiquement, évoquaient $T$. violaceum; dans ces souches existaient des chlamydospores intercalaires ou terminales et les aleuries étaient rares. D'autre part, deux souches de $T$. violaceum cultivées sur milieu enrichi à la thiamine ont développé en un mois un peu de duvet blanc qui portait quelques aleuries (photo 12) (pareil fait a déjà été publié par Georg en 1951 [16]) (20). Précisons encore que l'inoculation au cobaye des souches de $T$. gourvili à pousse lente a été négative, comme c'est la règle avec $T$. violaceum. Par contre, le comportement des diverses souches sur les milieux de Georg a toujours été différent : des $T$. gourvili à pousse lente étaient inhibés par la thiamine, qui stimulait au contraire la croissance des $T$. violaceum.

En définitive, en dépit de l'existence d'arguments permettant un rapprochement entre certaines souches de $T$. gourvili et de $T$. vio. laceum, il ne nous paraît guère justifié dans l'état actuel de nos connaissances de faire tomber $T$. gourvili (et $T$. soudanense, par

(19) La lésion pilaire déterminée étrít neo-endothrix; elle n'aboutissait pas a la formation de spores rondes bourrant le cheveu mais en restait au stade arthrosporé. Suivant les auteurs, $T$. currii posséderait périthèces et asques ; encore que la description de ces formations n'emporte pas la conviction, comme le faisaient déjà remarquer en 1923 Ota et Langeron [ 25 bis], les négliger purement et simplement dans la diagnose de ce champignon, comme nous l'avons fait en $1958[5]$, est sans doute une erreur.

(20) Les aleuries étaient nombreuses dans ces souches. 
voie de conséquence, compte tenu des conclusions du paragraphe précédent), en synonymie devant $T$. violaceum.

$5^{\circ}$ Nous avons considéré en $1957 T$. yaoundei, le principal agent des trichophyties camerounaises, comme distinct de $T$. violaceum pour les raisons suivantes : sur 390 cultures de Trichophyton (21) issus de lésions trichophytiques, nous avons isolé 241 fois $T$. yaoundei et nous n'avons pas cultivé une seule souche qui puisse s'étiqueter $T$. violaceum (22) ou former transition évidente entre le type yaoundei et le type violaceum. L'aspect macroscopique des colonies chocolat de $T$. yaoundei était souvent assez caractéristique (cratériforme ou vermiculé), de même que l'apparition fréquente dans les cultures de zones friables constituées par des files de chlamydospores se dissociant très facilement au moindre contact. Les cultures secondes, de pousse beaucoup plus facile, perdaient tout pigment. Nous avons récemment soumis T. yaoundei au test physiologique de L.-K. Georg et constaté qu'il était autotrophe, ce qui est une différence de plus avec $T$. violaceum, dont la croissance est stimulée par la thiamine. Cependant, nos souches sont isolées depuis trois ans; elles sont très modifiées et il est possible que leur comportement physiologique lui-même ait varié. Par ailleurs, il nous est arrivé à plusieurs reprises de voir se développer dans des primocultures des colonies dont la forme était primitivement très évocatrice de $T$. yaoundei, et qui se sont différenciées progressivement en $T$. soudanense typique. Enfin, il arrive fréquemment que le centre surélevé et creux de certaines colonies faviformes de ce dernier Dermatophyte montre à l'examen microscopique des analogies certaines avec la zone friable de $T$. yaoundei (23).

Pour toutes ces raisons, la position taxonomique de $T$. yaoundei reste actuellement incertaine et des recherches ultérieures conduites sur des souches fraîchement isolées seront nécessaires pour essayer de la préciser.

\section{Conclusion}

Nous nous sommes efforcés de préciser les affinités de quelques Trichophyton d'origine africaine et, en fonction de nos informations et de notre expérience actuelles, nous avons été conduits aux conclusions, sans doute provisoires, que voici :

(21) Nous avons isolé, à côté des souches de $T$. yaoundei, 149 souches de 7. soudanense.

(22) Nous avons étudié 1.050 souches de $T$. violaceum d'origine tunisienne.

(23) Ce type de colonie correspond peut-être à la souche T.A. 127 de Salazar Leite et Bastos da Luz (1950) [30] où ces auteurs notent la tendance du centre faviforme à constituer de nombreuses chlamydospores. 
$1^{\circ}$ Il y a peu d'arguments pour identifier T. ferrugineum à T. soudanense. Par contre, le déplacement de ce Dermatophyte du genre Microsporum au genre Trichophyton reste sujet à discussion.

$2^{\circ} T$. gourvili est très proche de $T$. soudanense, dont il représente un stade évolutif ; entre les deux espèces, les aspects intermédiaires sont parfois assez fréquents (au Tchad, par exemple). Dans ces conditions, on peut admettre la mise en synonymie de $T$. gourvili devant $T$. soudanense, à la condition toutefois de préciser lors des diagnoses la variété culturale, soudanense, gourvili, intermedium (quand il s'agit de cultures présentant des zones orangées et violettes), de la souche isolée.

$3^{\circ}$ Les Trichophyton blancs isolés du Tchad, et que nous avons considérés initialement comme des $T$. tonsurans, sont en réalité, selon toute vraisemblance, des aspects atypiques ou évolutifs de T. soudanense, que l'on pourrait qualifier de pseudotonsurans. Nous attirons l'attention sur les difficultés de leur diagnostic et sur les confusions éventuellés en Afrique Noire entre ces Trichophyton blancs et $T$. tonsurans.

$4^{\circ} T$. gourvili et $T$. violaceum doivent être distingués en dépit de quelques éléments de discussion en faveur de leur rapprochement; les deux champignons sont dans la règle assez différents et les formes de transition naturelles semblent rares.

$5^{\circ}$ Les affinités de $T$. yaoundei restent à préciser et nous n'osons même pas affirmer que ce Dermatophyte camerounais soit plus proche de $T$. violaceum que de $T$. soudanense.

En fait, notre expérience des Dermatophytes en général, des souches africaines en particulier, nous incite après bien d'autres auteurs à mettre l'accent sur leur étroite parenté et sur la difficulté et la fragilité de certaines déterminations spécifiques. En ce qui concerne les souches atypiques, l'intérêt consiste moins d'ailleurs à les faire entrer de gré ou de force sous une appellation préexistante ou à en créer inutilement une nouvelle à leur intention, que d'apprécier à quelle espèce classique elles sont apparentées. Quant aux grandes espèces qui sont bien connues, ce qu'il importe de préciser, nous semble-t-il, c'est leur filiation : les Ctenomyces saprophytiques constituent-ils, comme le voulait Langeron [22], le tronc d'origine de tous les Dermatophytes ? $T$. violaceum dérive-t-il de $T$. soudanense par l'intermédiaire de $T$. gourvili et $T$. tonsurans ? Ne constituerait-il pas un autre aboutissement du même $T$. soudanense par l'intermédiaire des Trichophyton blancs? T. yaoundei est-il un satellite de $T$. violaceum ou de $T$. soudanense? Quelles 
sont les affinités du polymorphe $T$. rubrum? $T$. schoenleini ne serait-il qu'un $T$. verrucosum parfaitement adapté à l'homme ? T. ferrugineum constitue-t-il un stade d'évolution faviforme de Microsporum canis ?... Autant de problèmes qu'il conviendrait de résoudre et pour lesquels les investigations morphologiques classiques et même la mise en œuvre de tests physiologiques nous paraissent incapables de conduire à des conclusions satisfaisantes. Il est peu probable que des travaux biochimiques apportent des arguments nouveaux de valeur, car le métabolisme de la plupart des Dermatophytes est insuffisamment actif (c'est du moins ce qui nous semble ressortir des publications que nous avons lues et de quelques essais déjà entrepris au laboratoire); il serait peut-être plus profitable de multiplier d'une part les cultures monospores en suivant l'exemple de Georg $\left[16^{\text {bis }}\right]$, et d'orienter d'autre part les recherches ultérieures vers l'étude de leurs fractions antigéniques en mettant à profit les techniques modernes et élégantes de l'immunochimie (méthode d'Ouchterlony [26] et immunoélectrophorèse de Grabar et Williams [18] qui, appliquées aux levures du genre Candida, nous ont donné des résultats préliminaires très encourageants.

\section{BIBLIOGRAPHIE}

1. Andrieu (S.), Capron (A.) et Sultan (G.), 1959. - Contribution à la connaissance des teignes de la République de Guinée. Remarquable fréquence des teignes associées (Microsporie et Trichophytie). Bull. Soc. Path. Exot., 52, 259.

2. ANSEL (M.), 1957. - Mycoses et champignons parasites de l'homme, Doin et $\mathrm{C}^{10}$ édit., Paris.

3. Bafza (M.), 1934. - Note statistique sur les teignes du Maroc. Ann. de Parasit. hum. et comp., 12, 405.

4. Biguet (J.), Havez (R.) eí Tran Van Ky, 1959. - Les possibilités d'application aux champignons pathogènes de la méthode d'Ouchterlony et de l'immunoélectrophorèse. C.R. Académie des Sciences, 249, 895.

5. Biglet (J.), Deblock (S.), Cochet (G.) et Ouadraogo (P.), 1958 et 1959. - Première contribution à l'étude des teignes en Haute-Volta. Revue des dermatophyties et des dermatophytes en Afrique Noire. Arch. Orig. Serv. Docum. C.N.R.S., 1958, $\mathrm{n}^{\circ} 367$ et Ann. Paras. hum. et comp., 1959, 34, 694.

6. Bieuet (J.), Ziegler (P.), Cochet (J.) et Duc (G.), 1959. — Première contribution à la connaissance des teignes de la République du Tchad (Moyen Chari : district de Koumra). Bull. Soc. Path. Exot., 52, 352.

7. Catanei (A.), 1933. - Description de Trichophyton gourvili n. sp., agent d'une teigne de l'homme. Bull. Soc. Path. Exot., 26, 377. 

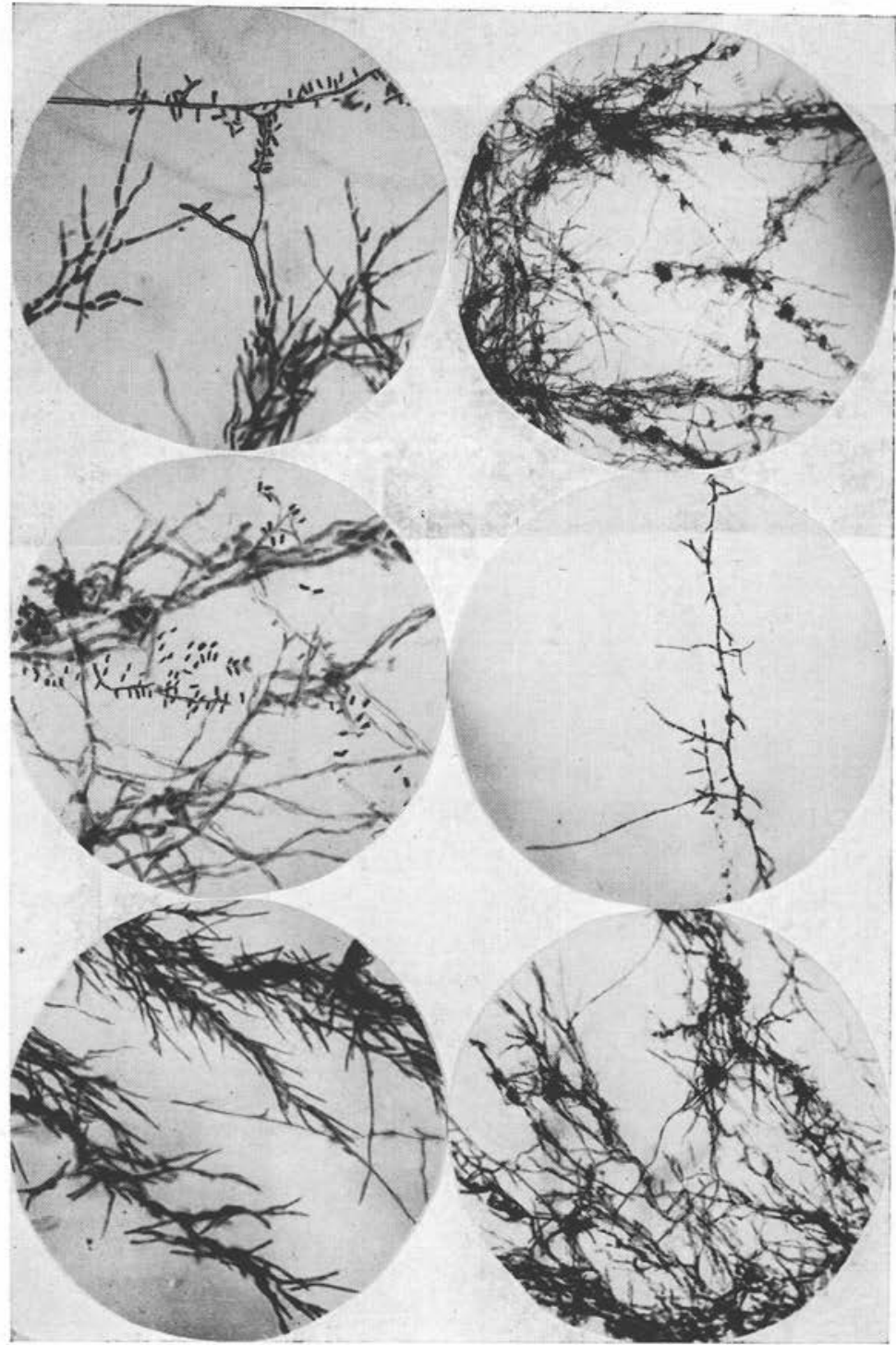

1. - Trichophyton soudanense : aspect du mycélium arthrosporé s'organisant en buissons et rameaux aériens portant des aleuries (1).

2. - Trichophyton gourvili: mycélium avec nodules nombreux.

3. - Trichophyton gourvili : même lame que précédemment mais à un grossissement supérieur qui montre les aleuries (1).

4. - Trichophyton gourvili : élément mycélien isolé qui montre la même organisation que $T$. soudanense (des rameaux poussant dans le sens opposé à celui de la eroissance du mycélium).

5. - Trichophyton soudanense se pigmentant de violet en vieillissant : l'aspect du mycélium reste typique de l'espèce soudanense.

6. - Trichophyton soudanense: autre culture de la même souche vieillie oủ les nodules sont apparus ; l'aspect évoque plutôt $T$. gourvili (cf. photo 2). 


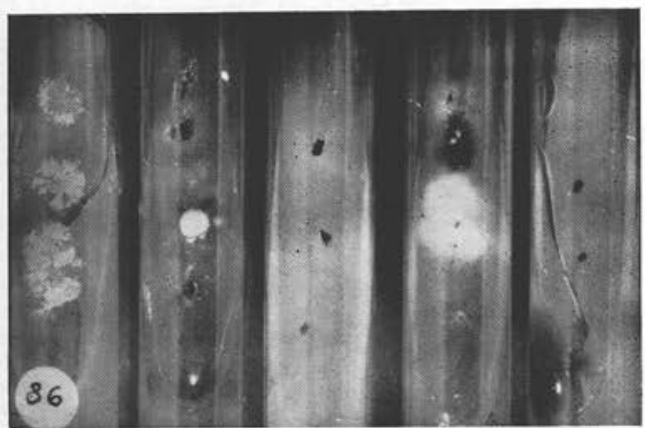

7. - Souche soudanense inhibée par la thiamine (1) (de gauche à droite, milieux : de Sabouraud $2 \%$, avitaminé, à la thiamine, à l'inositol, à la thiamine et à l'inositol).

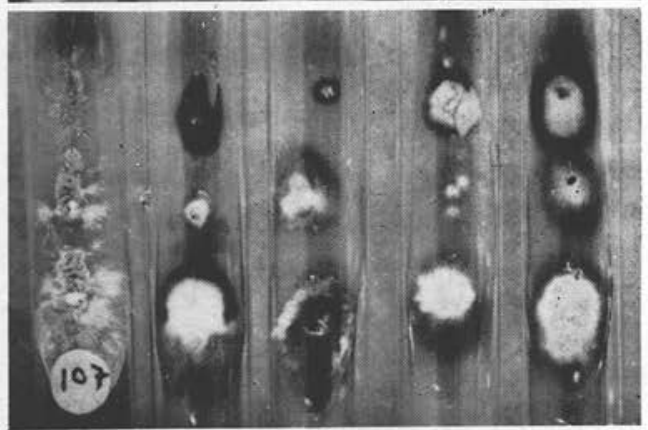

8. - Souche de T. soudanense non modifiée par la thiamine (2).

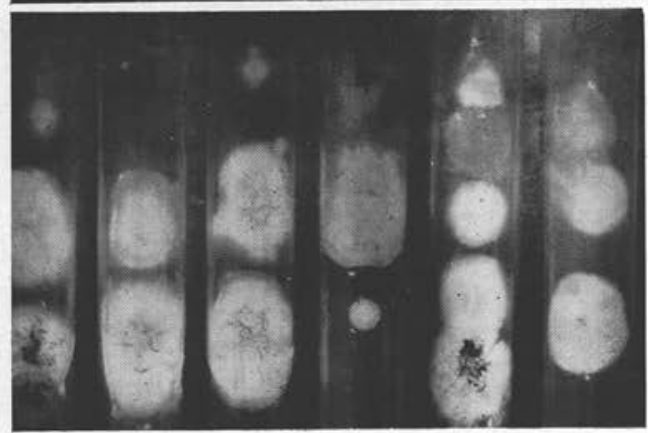

9. - Trichophyton soudanense blanes et $T$. tonsurans. Aspect macroscopique de 6 cultures dont les 4 de gauche correspondent à 2 souches de $T$. soudanense blancs et les 2 de droite à une souche de T.tonsurans. (Dans chaque groupe de 2 , le tube de gauche représente la primoculture, le tube de droite, une subculture après passage sur cheveux in vitro).

(1) Les hyphes sporifères des photographies 1,3 et 10 ont été soulignées à l'encre de Chine.

(2) Des images similaires sont fournies par certaines souches de T. gourvili. 


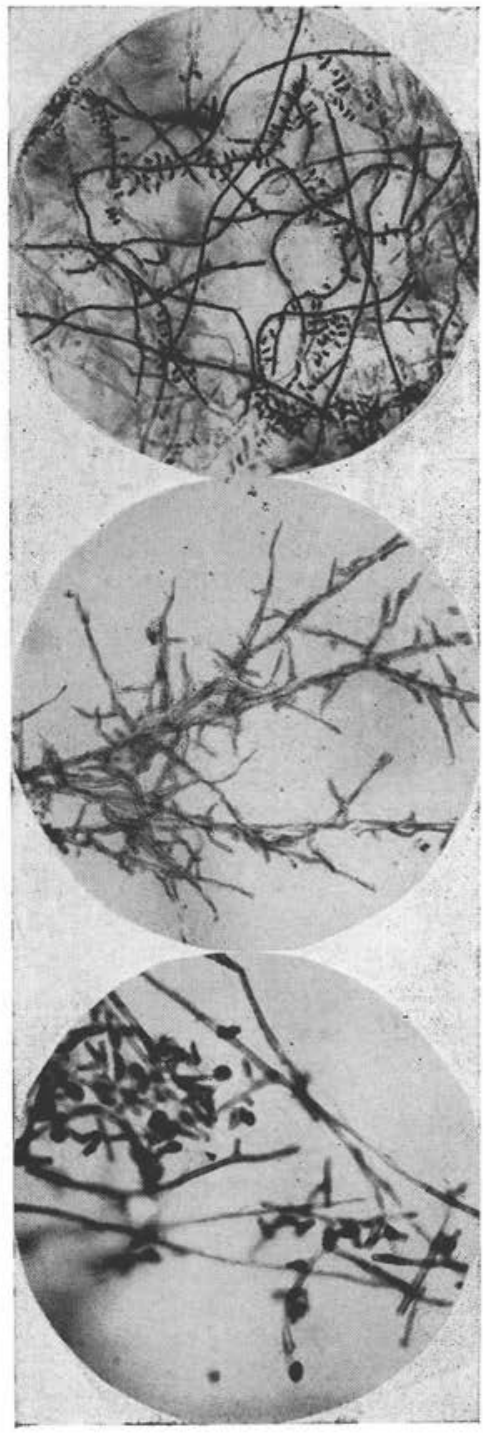

10. - Trichophyton soudanense blanc: mycélium avec très nombreuses aleuries (1).

11. - Trichophyton soudanense blanc: mycélium buissonnant avec rameaux opposés à son sens de progression.

12. - Trichophyton violaceum : quelques aleuries après culture sur milieu enrichi à la thiamine. 
8. Catanei (A.), 1937. - Description de deux nouvelles espèces et d'une variété nouvelle de champignons provoquant des teignes chez l'homme. Arch. Inst. Past. Algérie, 15, 265.

9. Catanel (A.), 1939. - Etudes des teignes du cuir chevelu dans les colonies françaises. Arch. Inst. Past. Algérie, 17, 47.

10. Catanei (A.), 1950. - Les teignes en Afrique du Nord. Répartition, formes cliniques et évolution. Champignons parasites. Maroc Médical, 29, 955.

11. Chalmers (A. J.) et Marshall (A.), 1914. - Tinea capitis tropicalis in the Anglo-Egyptian Sudan. Journ. Trop. Med. a. Hyg., 17, 257.

12. Cochet (G.), Doby-Dubois (M.), Deblock (S.), Doby (J. M.) et Vaiva (C.), 1957. - Contribution à la connaissance des teignes infantiles du Cameroun. Ann. Parasit. Hum. et Comp., 32, 580.

13. Conant (N. F.), Smith (D. T.), Baker (R. D.), Callaway (J. L.) et Martin (D. S.), 1945 et $1954 .-$ Manual of clinical mycology, $1^{\text {re }}$ édit. 1945, $2^{\circ}$ édit. 1954, W. B. Saunders Company Edit., Philadelphie et Londres.

14. Coutelen (F.), Cochet (G.), Biguet (J.), Mullet (S.), Doby-Dubois (M.) et Deblock (S.), 1956. - Contribution à la connaissance épidémiologique et mycologique des teignes infantiles de Tunisie. Ann. Paras. Hum. et Comp., 31, 449.

15. Deblock (S.), Andrieu (S.) et Chenal (C.), 1959. - Première contribution à la connaissance des teignes infantiles de la Côte-d'Ivoire (Afrique). Bull. Soc. Path. Exot., 52, 256.

16. Georg (L. K.), 1951. - The relation of nutrition to the growth and morphology of Trichophyton violaceum. II. The influence of nutritional factors on the morphology of $T$. violaceum. Mycologia, 43, 536 .

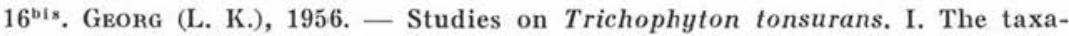
nomy of T. tonsurans. Mycologia, 48, 65 .

17. Geон (L. K.), 1957. - Dermatophytes, new methods in classification. Symposium on «Classification of the Dermatomycoses 》Eleventh International Congress of Dermatology, Stockholm.

18. Grabar (P.) et Williams (C. A.), 1953. - Method permetting the simutaneous study of electrophoretic and immunochemical properties of a proteins. Application to blood serum. Bioch. Biophys. Acta, 10, 193.

19. Grigoraki (L.), 1959. - Les bases d'un accord entre dermatologistes et mycologues sur une classification unique des dermatophytes. Mycopathologia et Mycologia applicata, 10, 317.

20. Joyeux (C.), 1912, - Sur le Trichophyton soudanense n. sp. C.R. Soc. Biol., $73,15$.

21. Joyeux (C.), 1914. - Contribution à l'étude des teignes africaines : Trichophyton soudanense. Arch. de Parasit., 16, 447.

22. Langeron (M.), 1945. - Précis de Mycologie, Masson et $\mathrm{C}^{10}$ édit., Paris.

23. Langeron (M.) et Vaubreuseghem (R.), 1952. - Précis de Mycologie, $2^{\circ}$ édition, Masson et $\mathrm{C}^{10}$ édit., Paris.

24. Lapidus (M.), 1950. - Les teignes en Tripolitaine. Flore et Distribution comparées des teignes nord-africaines. Thèse doctorat en médecine, Paris.

25. Ота (M.), 1922. - Sur deux espèces nouvelles de Dermatophytes en Mandchourie : Microsporum ferrugineum et Trichophyton pedis n. sp. Bull. Soc. Path Exot., 15, 588.

$25^{\text {bis }}$. Ota (M.) et Langeron (M.), 1923. - Nouvelle classification des Dermatophytes. Ann. de Parasit. Hum. et Comp., 1, 305. 
26. Ouchterlony (O.), 1949. - Antigen antibody reactions in gels. Acta Pathol. Microbiol. Scand., 26, 507.

27. PALDROK (H.), 1953. - On the variability and classification of dermatophytes. Acta Dermatologica Venereologica, 3, 1.

28. Rivalier (E.), 1954. - Description de Sabouraudites precox nova species, suivie de remarques sur le genre Sabouraudites, 86, 276.

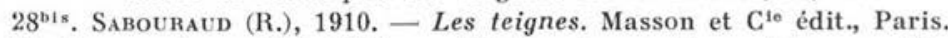

29. Salazar Leite (A.), 1958. - Tinhas dos nativos africanos. Sixth Intern. Congr. on Trop. Med. a. Malaria, Instituto de Medicina Tropica, Lisbonne, 137.

30. Salazar Leite (A.) et Bastos da Luz (I. V.), 1950. - Estudos sobre os agentes causais de tinhas en nativos africanos de raça negro. II. Congresso Luso-Espanhol de Dermatologia, Lisbonne.

31. Salazar Lette (A.), Bastos da Luz (J. V.) et de Merra (M. T. V.), 1947. Relatorio da Missão Medica do Instituto de Medicina Tropical a Angola en 1945. Anais Inst. Med. Trop., 465.

32. Salazar Leite (A.) et Re (L.), 1955. - Contribution à l'étude ethnologique des populations africaines. Archiv. Inst. Past. Algérie, 33, 344.

33. Sigalova (E. E.), 1951. - In Troyan (G. A.), 1958.

34. TAlice (R. V.), 1931. - Sur une souche de Trichophyton ferrugineum (Ota, 1921) (Microsporum ferrugineum Ota, 1921) isolée à Montevideo. Ann. de Parasit. Hum. et Comp., 9, 77.

35. Troyan (G. A.), 1956. — [Variabilité dirigée des Dermatophytes. Mikrobiol. Journ. Acad. Nauk, Rep. Soc. Sov. Ukrainienne, 20, 21] (en ukrainien).

36. Vanbreuseghem (R.), 1950. - Diagnose et systématique des Dermatophytes. Contribution à la connaissance des teignes du Congo Belge. Ann. Soc. Belge Méd. Trop., 30, 865.

37. Vanbreuseghem (R.), 1955. - Le Con̋̊o Belge et la mycologie médicale. Académie Royale des Sciences Coloniales, Classe des Sciences Naturelles et Médicales. Mémoires, $1,1$.

38. Vanbreuseghem (R.) et Van Brussel (M.), 1952. - La terre, facteur de mutation d'un dermatophyte : Langeronia soudanensis (Joyeux, 1912) Vanbreuseghem, 1950. Ann. Soc. Belge Méd. Trop., 32, 79.

39. Zimerinov (A. A.), Besnos (T. I.) et Rafolovitch (C. M.), 1953. - In Troyan (G. A.), 1958.

(Travail du Laboratoire de Parasitologie

de la Faculté de Médecine et Pharmacie de Lille; Prof. J. Biguet)

\section{ERRATUM}

(Article J. Biguet et coll., ces Annales, n* 5-6, 1959, p. 696). - C'est par erreur que $T$. gourvili figure en Guinée sur la carte de distribution des dermatophytes africains. Jusqu'ici, la présence de cette espèce n'a pas été signalée dans ce territoire. 